\title{
Anterior megalophthalmos - A case report
}

\author{
Dr. Dipti N Kale ${ }^{1,}$ Dr Naresh Kumar Singh ${ }^{2}$ \\ ${ }^{1,2}$ (Department of ophthalmology, Regional institute of medical sciences, Imphal)
}

\begin{abstract}
We describe a 10 years old male who suffered from diminution of vision and raised intra ocular pressure (left eye was more severely affected than the right), the use of some ophthalmologic examinations to evaluate the anatomic structures and visual functions of both eyes. Slit lamp and biometric examinations showed very large corneas, very deep anterior chambers, short vitreous length and miotic left pupil. Gonioscopy showed excessive mesenchymal tissue in the angle of the anterior chamber. Corneal transparency, corneal thickness and lens were normal. Because the patient had bilateral megalocornea, raised IOP, deep anterior chamber and atrophic changes in the iris, anterior megalophthalmos was diagnosed. The case was studied in detail as the condition is rare. Secondary effects such as iridodonesis, miosis, atrophy of the iris stroma, lens subluxation and occurrence of cataractous lens should be kept in mind. The importance of biometric data for diagnosis and differential diagnosis in primary infantile glaucoma and other diseases with megalocornea is discussed.
\end{abstract}

Keywords: gonioscopy, infantile glaucoma, meegalocornea, megalophthalmos, raised IOP

\section{Introduction}

A ten year old male child was brought to the OPD by the mother with chief complaints of blurring of vision, watering from eyes, pain in eyes and colored halos since the last one year. The blurring of vision was gradual in onset and progress. The eyes were noted to be large right from birth by the mother but no treatment was sought. There is no previous history of spectacle use or any other significant history. The father and elder brother of the patient were blind. Both had gradual loss of vision over some months. They were not available for examination.

\section{Ophthalmologic Examinations}

On Ophthalmologic Examination there was gross obvious enlargement of both the corneas. Thereafter various other Occular Examinations were undertaken, the findings of the same are as follows:

1. Both the corneas were transparent.

2. Corneal thickness was measured by pachymetry and was found to be near normal.

3. The corneal curvature was measured by keratometry and was found to be normal (K reading: right eye43.25/42.75, left eye- 43.00/43.75).

4. The most significant finding in the cornea was the enlarged corneal diameter which was measured with the help of calipers. In the right eye the corneal diameter was $13.5 \mathrm{~mm}$ and in the left eye it was $15.5 \mathrm{~mm}$.

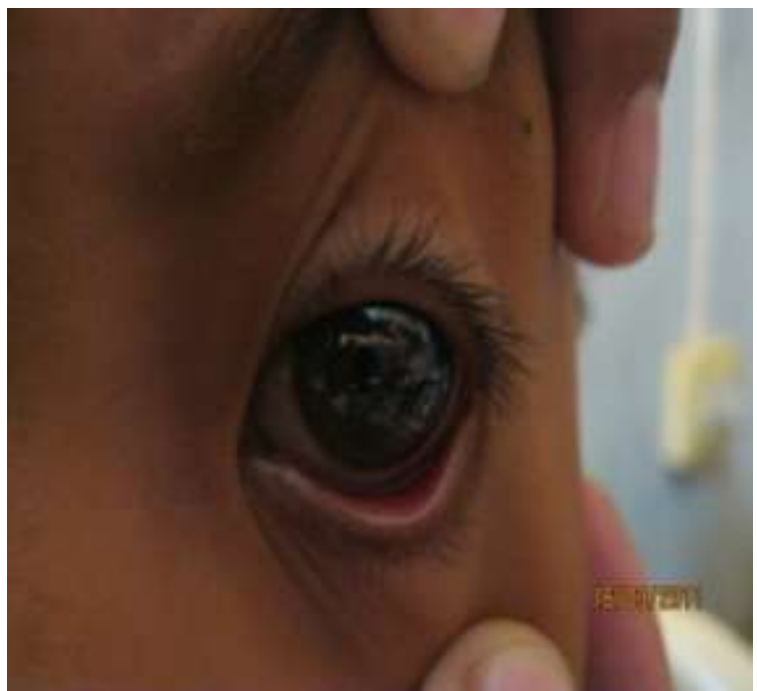

Fig. 1

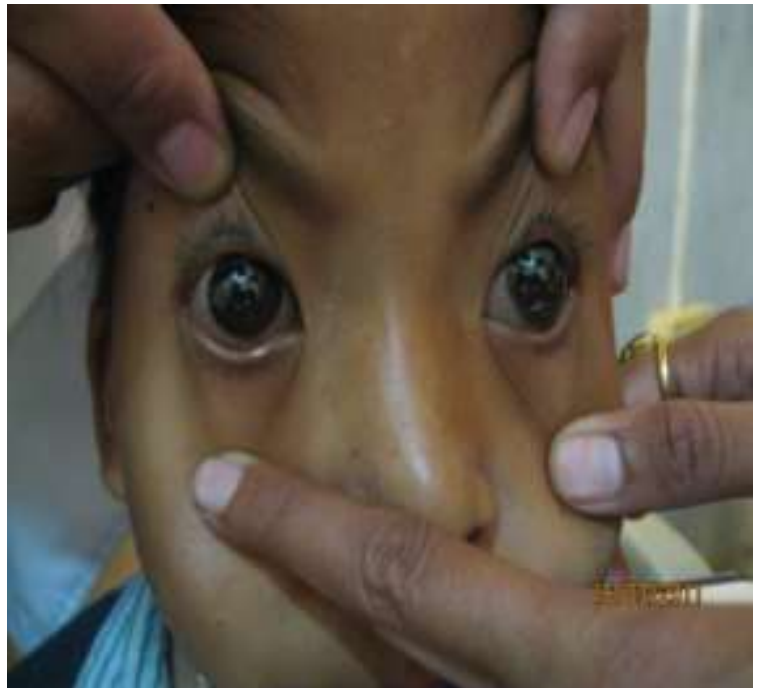

Fig. 2 


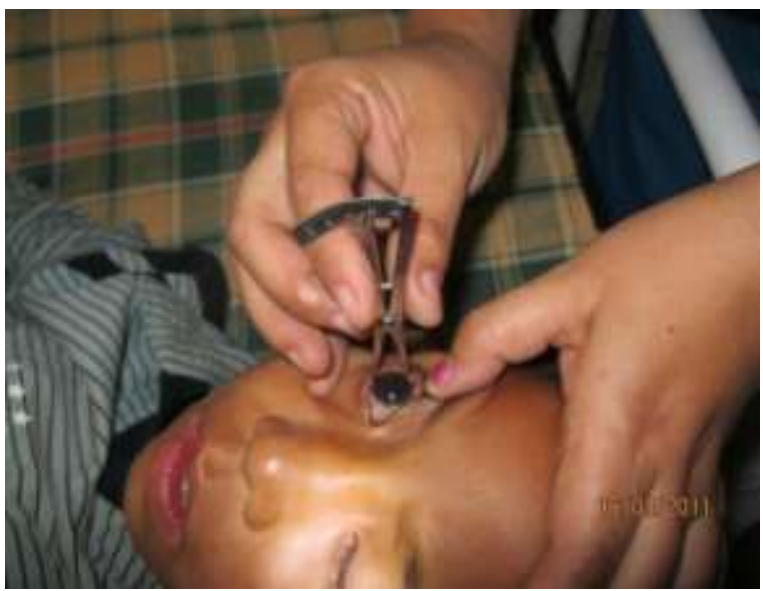

Fig. 3

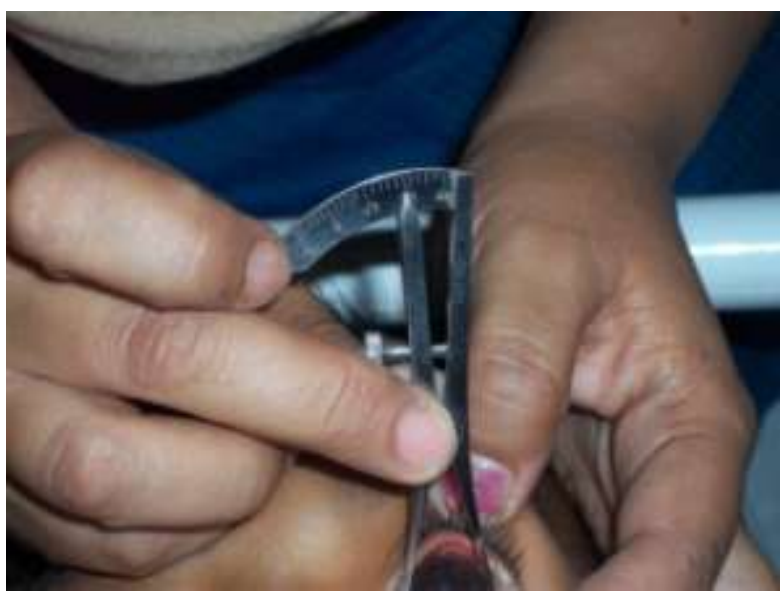

Fig. 4

\section{A - Scan Biometry:}

Biometry showed the axial length to be increased to $24.93 \mathrm{~mm}$ in the right eye and $26.56 \mathrm{~mm}$ in the left eye. Also the depth of the anterior chamber was increased in both eyes to $5.68 \mathrm{~mm}$ and $6.02 \mathrm{~mm}$ for right and left eye respectively. Lens thickness was $3.31 \mathrm{~mm}$ for right eye and $3.53 \mathrm{~mm}$ for left eye. Vitreous length was $16.6 \mathrm{~mm}$ in the right eye and $15.86 \mathrm{~mm}$ in the left eye. Vitreous index was $64.82 \%$ for right eye and $59.71 \%$ for left eye.

\section{Slit Lamp Examination:}

Pupil size was $3 \mathrm{~mm}$ in the right eye and $2 \mathrm{~mm}$ in the left eye i.e. miotic left pupil Iris was normal in appearance with the naked eye. Slit lamp examination showed clear corneas, enlargement of the complete anterior segment of the eyes and the left iris showed atrophic patches on the superior and temporal margins.

Gonioscopy was done in both the eyes but the angle structures were not clearly visible and could not be well differentiated. There was evidence of excessive mesenchymal tissue in the angle. The intraocular pressure in the right eye was $31.8 \mathrm{~mm} \mathrm{Hg}$ and in the left eye was $34.4 \mathrm{~mm} \mathrm{Hg}$ with applanation tonometry. Treatment was started for raised IOP with dorzolamide and timolol maleate $0.5 \%$ eye drop. Vision in the right eye was $6 / 9$ and in the left eye was 6/60. Myopic correction was $-0.5 \mathrm{D}$ for the right eye and $-3 \mathrm{D}$ for the left eye after which vision improved to $6 / 6$ in the right eye and 6/36 in the left eye. Lens and fundus appeared to be normal.

On follow up IOP was found to be normal. Patient was continued on anti glaucoma treatment and reviewed every month. Unfortunately chromosomal analysis could not be done for the patient.

\section{Discussion}

The new born cornea measures about $10 \mathrm{~mm}$ in horizontal diameter and reaches the average adult measurement of $11.75 \mathrm{~mm}$ by 2 yrs of age. Megalocornea is present if the horizontal diameter of a new born cornea is $12 \mathrm{~mm}$ or more and if adult cornea is $13 \mathrm{~mm}$ or more.

An enlarged cornea occurs in three patterns:-

1. Megalocornea unassociated with other ocular abnormalities.

2. Anterior megalophthalmos, that consists of megalocornea, iris and angle abnormalities.

3. Buphthalmos in infantile glaucoma ${ }^{1}$.

Anterior Megalophthalmos is a rare $\mathrm{X}$ linked recessive disease ${ }^{2}$, which manifests as bilaterally symmetrically enlarged clear corneas that remain stable (non progressive) throughout life and sometimes contain a stromal mosaic pattern ${ }^{1}$. This condition predisposes the patient to glaucoma ${ }^{3}$. The patients present a very deep anterior chamber and a vitreous index (vitreous length/axial length into 100) below $69 \%{ }^{4}$.

The iridocorneal angle is open but contains excess mesenchymal tissue, whereas iris manifests a hypoplastic anterior stroma and transillumination defects ${ }^{1}$. Secondary effects such as iridodonesis, miosis, atrophy of the iris stroma and cataractous lens should be kept in mind ${ }^{2}$.

According to Peter Laibson and George Waring, the two associations that threaten vision are the frequently elevated IOP, which requires annual lifelong examination for early detection, and cataracts which often appear in the fourth decade ${ }^{1}$.

If bilateral clear corneas of normal thickness measure $13 \mathrm{~mm}$ or more in diameter without associated ocular abnormalities, the non progressive disorder of Simple Megalocornea exists and once the diagnosis is clearly made no follow up is necessary ${ }^{1}$. 
Buphthalmos is a term to describe the abnormal progressive enlargement of an infant's eye secondary to elevated IOP; usually noticed in the first year of life. The classic triad of findings usually seen is epiphora, photophobia and blepharospasm which result from corneal edema, often with associated breaks in the Descemet membrane called Haab striae.

Additional non specific signs of glaucoma in early life include a deep anterior chamber and optic nerve cupping ${ }^{5}$. Also in contrast to megalocornea, congenital glaucoma is marked by the absence of transillumination defects, absence of pigment dispersion and less pronounced enlargement of the anterior chamber.

\section{Conclusion}

The differentiation between simple megalocornea, megalophthalmos anterior and primary infantile glaucoma is often difficult, but very important, as in the latter case surgical treatment prevents blindness.

\section{References}

[1] Robinson DH. Peter R Laibson, George O Waring, Editor. Diseases of the Cornea In: Paediatric Ophthalmology $2^{\text {nd }}$ ed; (West Washington Square Philadelphia, WB Saunders Company1983);468-470

[2] Tsai CK, Lai IC, Kou HK, Tong MC, Fang PC. Chang Gung Med J 2005 Mar;28(3):191-5

[3] Roche O, Dureau P, Uteza Y, Dufier JL. Congenital Megalocornea. J Fr Ophthalmol.2002 Mar;25(3):312-8

[4] Miere FM, Dellman JW. Biometry in X linked megalocornea pathognomonic findings: Br J Ophthalmol 1994;78:781-85

[5] Allingham RR, Damji FK et al. Childhood glaucomas classification and examination. Shields Text Book Of Glaucoma $6^{\text {th }}$ ed;(New Delhi,Walters Kluwer (India) Pvt Ltd 2011);206-208 